\title{
Schisandra fructus extract ameliorates doxorubicin-induce cytotoxicity in cardiomyocytes: altered gene expression for detoxification enzymes
}

\author{
Eun Hye Choi $\cdot$ Nari Lee $\cdot$ Hyun Jung Kim • \\ Mi Kyung Kim · Sung-Gil Chi · Dae Young Kwon · \\ Hyang Sook Chun
}

Published online: 15 November 2007

(C) Springer-Verlag 2007

\begin{abstract}
The effect of Schisandra fructus extract (SFE) on doxorubicin (Dox)-induced cardiotoxicity was investigated in H9c2 cardiomyocytes. Dox, which is an antineoplastic drug known to induce cardiomyopathy possibly through production of reactive oxygen species, induced significant cytotoxicity, intracellular reactive oxygen species (ROS), and lipid peroxidation. SFE treatment significantly increased cell survival up to $25 \%$, inhibited intracellular ROS production in a time- and dosedependent manner, and inhibited lipid peroxidation induced by Dox. In addition, SFE treatment induced expression of cellular glutathione $S$-transferases (GSTs), which function in the detoxification of xenobiotics, and endogenous toxicants including lipid peoxides. Analyses of 31,100 genes using Affymetrix cDNA microarrays showed that SFE treatment up-regulated expression of genes involved in glutathione metabolism and detoxification [GST theta 1 , mu 1 , and alpha type 2 , heme oxygenase 1 (HO-1), and microsomal epoxide hydrolase $(\mathrm{mEH})]$ and energy metabolism [carnitine palmitoyltransferase-1 (CPT-1), transaldolase, and transketolase]. These data indicated that SFE might increase the resistance to cardiac cell injury
\end{abstract}

E. H. Choi · N. Lee · H. J. Kim · D. Y. Kwon · H. S. Chun ( $₫)$ Food Safety Research Center, Korea Food Research Institute, 516, Backhyun, Bundang-gu, Sungnam, Kyonggi-do 463-746, South Korea e-mail: hschun@kfri.re.kr

\section{K. Kim}

Department of Food and Nutritional Sciences, Ewha Womans University, Seoul 120-750, Republic of Korea

S.-G. Chi

School of Life Sciences and Biotechnology,

Korea University, Seoul 136-713, Republic of Korea by Dox, at least partly, together with altering gene expression, especially induction of phase II detoxification enzymes.

Keywords Cardiomyocytes - Cytoprotection . Detoxification · Doxorubicin · Glutathione $S$-transferase * Schisandra fructus

\section{Introduction}

Schisandra fructus (SF), the fruit of the Schisandra chinensis Baillon, is a well-known traditional herbal medicine that is widely used as a stimulant, a sedative, an antitussive, and a tonic agent in China, Japan, and Korea [9]. It has been reported that SF has various pharmacological activities, including antihepatotoxic [12, 27], anti-inflammatory [14], anticarcinogenic [19], antioxidant, and detoxificant effects $[12,16]$. SF contains various active compounds, including essential oils, organic acids, tannins, anthocyanins and lignans [11].

Several in vitro and in vivo studies have shown that SF has potent antioxidative properties such as inhibition of lipid peroxidation, induction of the antioxidant system and scavenging of reactive oxygen species (ROS). Many studies also suggest that SF lignans protect hepatocytes and cortical cells against oxidative damage. These observed cytoprotective effects have been attributed to improvement of the glutathione (GSH) defense system and inhibition of cellular peroxide formation.

Doxorubicin (Dox) is an anthracycline antibiotic that is one of the most effective and widely used anticancer drugs. However, the clinical use of Dox has been limited as its use has been associated with the development of life-threatening cardiomyopathy and congestive heart 
failure $[4,20,23]$. Although Dox-induced myocardial dysfunction is multifactorial, the putative main mechanism for Dox-induced cardiotoxicity is the production of free radicals during its intracellular metabolism. Free radicals cause diverse oxidative damage to critical cellular components and membranes in heart tissues [6, 7, 17]. Moreover, the heart is very sensitive to oxidative stress owing to its highly oxidative metabolism, and it has a lower level of antioxidant defense systems than the liver [5].

Considerable efforts have been made to investigate the use of antioxidants to reduce the side effects of Dox administration. In our previous study, we found that anthocyanin, one of the antioxidant components of SF, reduced 5-fluorouracil-induced myelotoxicity [3], Doxinduced cytotoxicity, intracellular ROS production and lipid peroxidation in cardiomyocytes [2]. SF lingans also have been reported to increase Hsp25/70 expression levels and inhibit nuclear factor- $\kappa \mathrm{B}(\mathrm{NF}-\kappa \mathrm{B})$ activation.

Therefore, we investigated the protective effect of Shisandra fructus extract (SFE) on Dox-induced cytotoxicity and its antioxidative properties in $\mathrm{H} 9 \mathrm{c} 2$ cardiomyocytes. The SFE-regulated gene expression in the Dox-induced cardiotoxicity system was further characterized using cDNA microarray techniques, which allowed us to systematically understand the cardioprotective mechanisms of SFE at the whole-genome level.

\section{Materials and methods}

\section{Extraction of Schizandra fructus}

Dried fruits of Schizandra chinensis were extracted for $3 \mathrm{~h}$ with $80 \%$ ethanol by using a reflux apparatus to yield the extract after removal of the solvent in vacuo. The ethanol extract was then resolubilized in water and semi-purified by a solid-phase extraction (SPE) with a C18 cartridge (Waters Corp., Milford, MA). The final eluant (SFE) was freeze-dried and resolubilized in phosphate-buffered saline (PBS) for subsequent assays.

\section{Cell culture}

H9c2 myocardial cells, spontaneously immortalized ventricular myoblasts of rat embryo, were purchased from the American Type Culture Collection (Manassas, VA). Cells were cultured in Dulbecco's modified Eagle's medium (DMEM) supplemented with $10 \%$ fetal bovine serum (FBS) at $37^{\circ} \mathrm{C}$ in $5 \% \mathrm{CO}_{2}$. Cell culture medium and supplements were purchased from GibcoBRL (Grand Island, $\mathrm{NY}$ ). The medium was changed every 2-3 days.
Cell viability

Sub-confluent cells were trypsinized and seeded onto 96well plates at a density of $1.5 \times 10^{5}$ cells $/ \mathrm{ml}$ and incubated for $24 \mathrm{~h}$ before treatment. Thereafter, cells were exposed to $1 \mu \mathrm{M}$ Dox for $24 \mathrm{~h}$ and then incubated in fresh medium with SFE at a concentration of $30-1,500 \mu \mathrm{g} / \mathrm{ml}$ as a gallic acid equivalent (GAE) for a further $24 \mathrm{~h}$. The effects of SFE on Dox-induced cytotoxicity were assessed using the sulforhodamine B (SRB) assay, as previously described [2, 21]. In brief, after fixation of the cells by the addition of $50 \%$ trichloroacetic acid (TCA) solution, the plate was stained with $0.4 \%$ SRB solution, and excess dye was then washed out. The unwashed dye was eluted and quantified spectrophotometrically at $550 \mathrm{~nm}$ using a microplate reader (Molecular Devices, Sunnyvale, CA). Cell viability was determined as the percentage of surviving cells as compared with that of the Dox-treated control.

\section{Intracellular ROS}

Intracellular ROS induced by Dox was measured using $2^{\prime} 7^{\prime}$ dichlorodihydrofluorescein diacetate $\left(\mathrm{H}_{2} \mathrm{DCFDA}\right.$, Molecular Probes, Eugene, OR) by the method of Wang and Joseph [24] with slight modifications. H9c2 cells were loaded with $20 \mu \mathrm{M} \mathrm{H}_{2}$ DCFDA for $10 \mathrm{~min}$, followed by two washes with HBSS. The dichlorodihydrofluorescein (DCF) fluorescence was detected using a fluorescence spectrophotometer (BMG LABTECH GmbH, Offenburg, Germany) with the excitation of $485 \mathrm{~nm}$ and emission of $520 \mathrm{~nm}$, and fluorescence image was visualized by a fluorescence microscope.

\section{Lipid peroxidation}

The thiobarbituric acid reactive substances (TBARS) method was used to determine the effect of SFE on Doxinduced lipid peroxidation according to the method of Jo and Ahn [13] with slight modification. H9c2 cells were washed twice with PBS and lysed in sodium dodecyl sulfate (SDS) solution. The $200 \mu \mathrm{l}$ of cell lysates were transferred to test tubes and $50 \mu \mathrm{l}$ of butylated hydroxytoluene (BHT) was added to prevent sporadic lipid peroxidation during heating. Next, a solution of $1.5 \mathrm{ml}$ of $0.5 \mathrm{M} \mathrm{HCl}, 1.5 \mathrm{ml}$ of $20 \mathrm{mM}$ TBA and distilled water was added. The reaction mixture was well mixed and then heated for $30 \mathrm{~min}$ in a boiling water bath. After cooling for $20 \mathrm{~min}, 2 \mathrm{ml}$ of $n$-butanol was added. The mixture was mixed vigorously and centrifuged at 3,000 rpm for $15 \mathrm{~min}$. The fluorescence of the organic layer was measured through a fluorescence spectrophotometer with an excitation of $515 \mathrm{~nm}$ and emission of $555 \mathrm{~nm}$. TBARS levels were calculated from a 
standard curve using 1,1,3,3-tetramethoxypropane and normalized to the protein content.

\section{Glutahione $S$-transferase activity}

GST activity was assayed spectrophotometrically at $340 \mathrm{~nm}$ using the standard substrate 1-chloro-2, 3-dinitrobenzene (CDNB) and co-substrate GSH by the method of Habig et al. [8]. The increase rate of absorbance at $340 \mathrm{~nm}$ is directly proportional to GST activity. The activity was calculated using an extinction coefficient of $9.6 \mathrm{mM}^{-1}$ $\mathrm{cm}^{-1}$ and expressed as nmol of CDNB-GSH conjugate formed per min per mg protein.

\section{Isolation of total RNA and microarray analysis}

The total RNA from the H9c2 cells was isolated using TRIzol (Invitrogen, Carlsbad) and then cleaned using the RNeasy Mini Kit (Qiagen $\mathrm{GmbH}$, Hilden, Germany) according to the manufacturer's instructions. Probe synthesis from total RNA (5 $\mu \mathrm{g})$, hybridization, detection and scanning was performed according to the standard protocols from Affymetrix (Affymetrix Inc., Santa Clara, CA). Briefly, cDNA was synthesized from total RNA using the one-cycle cDNA Synthesis Kit and T7-oligo (dT) primers. Double-stranded cDNA was used for in vitro transcription (IVT). cDNA was transcribed using the GeneChip IVT Labeling Kit (Affymetrix), and 10-15 $\mu \mathrm{g}$ of labeled cRNA was fragmented to form $35-200 \mathrm{bp}$ fragments. After hybridization to the Rat 2302.0 gene chips (Affymetrix), according to the Affymetrix standard protocol, the arrays were washed and stained with a streptavidin-phycoerythrin complex, and the intensities were determined using a GeneChip scanner 3000 (Affymetrix) and controlled by GCOS Affymetrix software 1.4 (Affymetrix). After data normalization, a DNA microarray data analysis was performed using GenPlex ${ }^{\mathrm{TM}}$ (Istech, Korea). The detectable expressed genes were defined using P-, M-, and A-calls according to the Affymetrix algorithm and intensity of the genes. We selected only those genes with at least four P-calls. Cross comparisons of the control versus treated data from each experiment were analyzed on the filtered probes. Only genes that were statistically significant and were up- or down-regulated by at least twofold compared with the control were judged as valid and identified as differentially expressed genes (DEGs).

\section{Statistical analysis}

All cytochemical data were given as mean \pm SEM. Statistical analysis was carried out using the Student's $t$ test.
Differences with $p<0.05$ were accepted as statistically significant. For microarray data, signal intensities between two groups were analyzed by the Welch's $t$ test with a significance level of $p<0.05$.

\section{Results}

Modulation of Dox-induced cellular response in $\mathrm{H} 9 \mathrm{c} 2$

Treatment of H9c2 cardiomyocytes with Dox and SFE affected cellular responses including cell viability, intracellular ROS production, lipid peroxidation and GST activity.

Dox-induced significant cytotoxicity and the halfmaximal inhibitory concentration $\left(\mathrm{IC}_{50}\right)$ of Dox on the growth of $\mathrm{H} 9 \mathrm{c} 2$ cells was determined to be $\sim 1 \mu \mathrm{M}$. Thus, this concentration was chosen for all further experiments except the intracellular ROS analysis. Figure 1 shows that SFE has a protective effect against Dox-induced cytotoxicity. The addition of 150 or $300 \mu \mathrm{g} /$ $\mathrm{ml}$ SFE significantly increased cell survival by 15 and $25 \%$, respectively, compared with the Dox-treated control cells $(p<0.05)$.

The effects of Dox and/or SFE on the intracellular ROS formation are shown in Fig. 2. The DCF fluorescence was proportional to the amount of intracellular ROS formed. Cells exposed to Dox showed an increase in DCF fluorescence in a time-dependent manner, whereas in cells

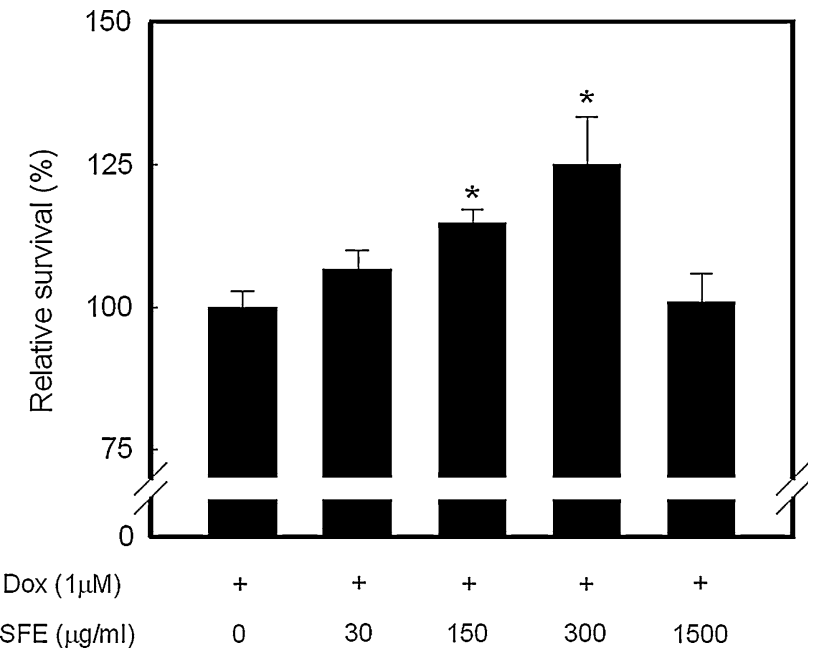

Fig. 1 Cytoprotective activities of SFE on Dox-induced cytotoxicity in $\mathrm{H} 9 \mathrm{c} 2$ cardiomyocytes. After $24 \mathrm{~h}$ exposure to $1 \mu \mathrm{M}$ Dox, cells were incubated with SFE for a further $24 \mathrm{~h}$ and then assessed for cytotoxicity using the SRB assay. Cell survival is represented by a percentage of cell viability compared with the Dox-treated control. The concentration of SFE was expressed as a gallic acid equivalent (GAE). ${ }^{*} p<0.05$ compared with the Dox-treated control 
Fig. 2 Scavenging effect of SFE on Dox-induced intracellular ROS formation in H9c2 cardiomyocytes. Production of intracellular ROS was determined by measurement of DCF fluorescence, which was emitted by intracellular oxidation. a After 1 and $10 \mathrm{~h}$ incubation with $10 \mu \mathrm{M}$ Dox and SFE, the fluorescence was measured with a fluorescence microplate reader. DCF fluorescence is expressed as \% increase in DCF fluorescence with respect to the intensity at 0 time. ${ }^{* * *} p<0.01$ and ${ }^{* * *} p<0.001$ compared with the Dox-treated control. b Fluorescence images show the ROS levels in control cells and the cells stimulated with Dox or Dox and SFE a

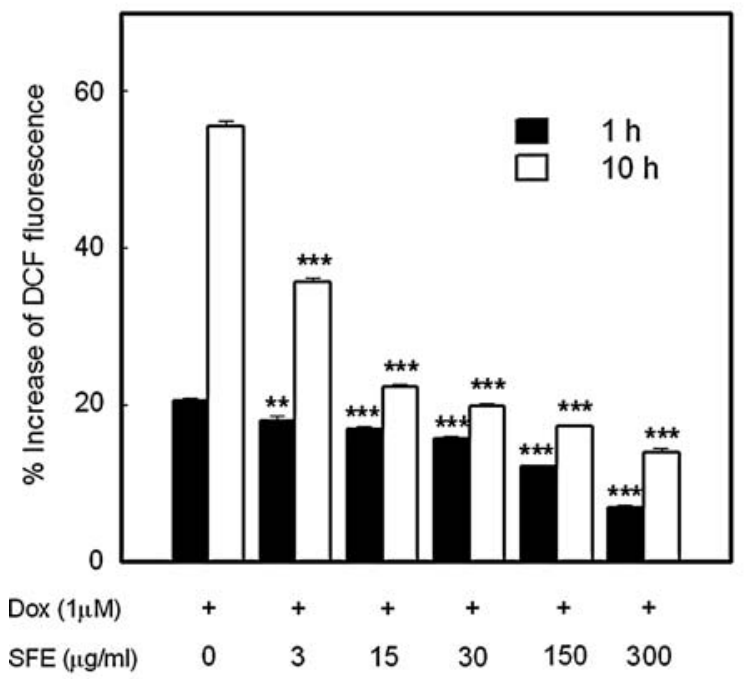

b

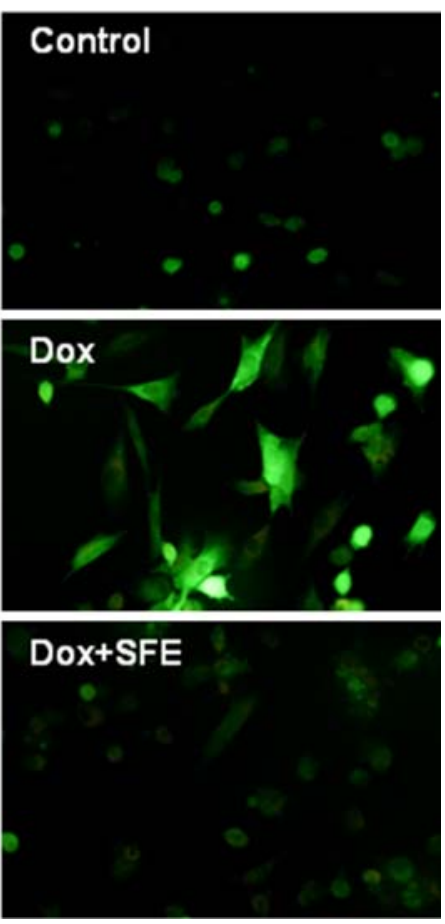

treated with SFE for $1 \mathrm{~h}$, the DCF fluorescence significantly reduced in a dose-dependent manner, even at the lowest concentration tested $(3 \mu \mathrm{g} / \mathrm{ml})$. After $10 \mathrm{~h}$ of incubation time, the percentage increase in DCF fluorescence widened the gap between Dox-treated control and SFEtreated cells.

ROS initiate the autocatalytic chain of lipid peroxidation, resulting in the formation of a variety of toxic species and, ultimately, cell death. Thus, lipid peroxidation has been recognized as a potential mechanism of cell injury. Lipid peroxidation, as measured by the TBARS levels after $24 \mathrm{~h}$ exposure of $\mathrm{H} 9 \mathrm{c} 2$ cells to $1 \mu \mathrm{M}$ of Dox, was significantly higher by about $30 \%$ compared with the Doxuntreated control $(p<0.01)$. As shown in Fig. 3, treatment with $300 \mu \mathrm{g} / \mathrm{mL}$ SFE treatment significantly reduced the TBARS level to a similar level as that of Dox-untreated control $(p<0.05)$; however, a low concentration of SFE $(30 \mu \mathrm{g} / \mathrm{ml})$ did not have a significant effect on TBARS production.

We also analyzed the activity of cellular GSTs of which the main function is to catalyze the conjugation of electrophilic xenobiotics or their metabolites, including peroxides; the cellular GST activity is shown in Fig. 4, and it can be seen that Dox treatment did not significantly induce GST activity. Treatment with $30 \mu \mathrm{g} / \mathrm{ml}$ SFE increased GST activity significantly by 18 and $24 \%$, respectively, compared with the Dox-treated and untreated controls. However, $300 \mu \mathrm{g} / \mathrm{ml}$ SFE showed a slight increase in GST activity compared with the Dox-treated and untreated controls.

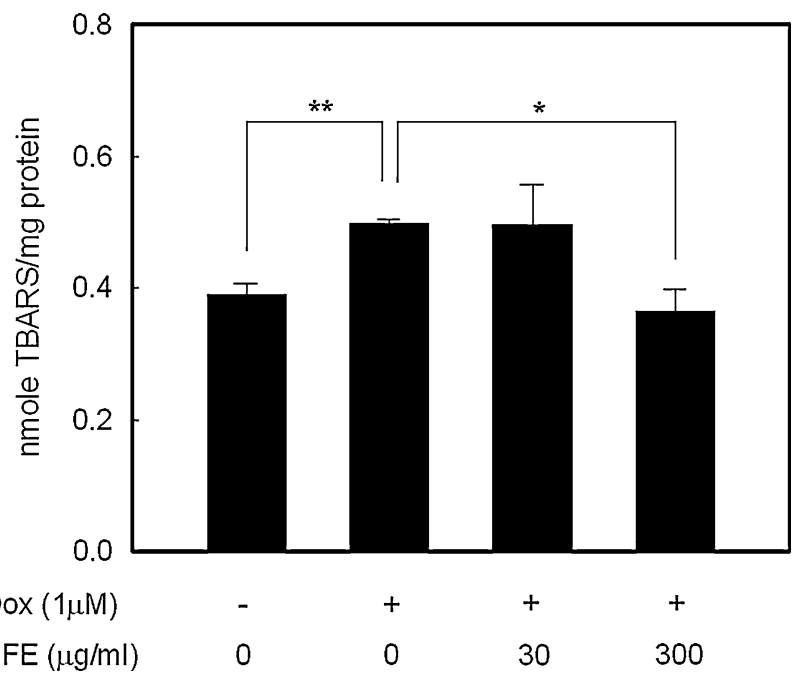

Fig. 3 Effect of SFE treatment on Dox-induced lipid peroxidation in H9c2 cardiomyocytes. After $24 \mathrm{~h}$ exposure to $1 \mu \mathrm{M}$ Dox, cells were incubated with SFE for a further $24 \mathrm{~h}$, and then assessed for lipid peroxidation using the TBARS method. $* p<0.05$, $* * p<0.01$

Altered gene expression by Dox and SFE treatment in $\mathrm{H} 9 \mathrm{c} 2$

The numeric distribution of total genes and the differentially expressed genes (DEG) treated with $1 \mu \mathrm{M}$ of Dox and/or $300 \mu \mathrm{g} / \mathrm{mL}$ of $\mathrm{SFE}$ in $\mathrm{H} 9 \mathrm{c} 2$ cardiomyocytes are shown in Fig. 5. Dox and/or SFE treatment in $\mathrm{H} 9 \mathrm{c} 2$ cardiomyocytes caused significant changes in gene expression. A total of 568 genes (151 were up-regulated, 


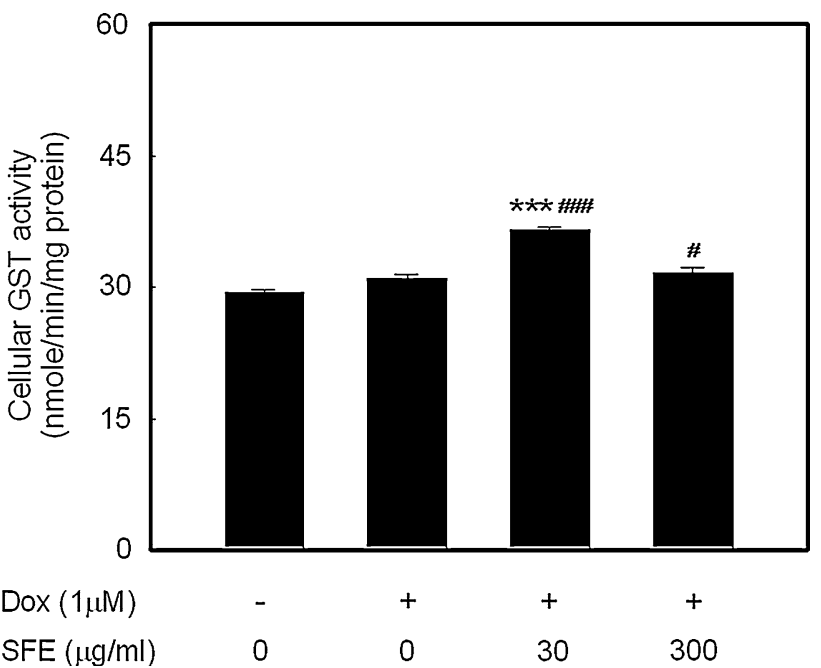

Fig. 4 Effect of SFE treatment on the GST activity in H9c2 cardiomyocytes. After $24 \mathrm{~h}$ exposure to $1 \mu \mathrm{M}$ Dox, cells were incubated with SFE for a further $24 \mathrm{~h}$, and GST activities were then

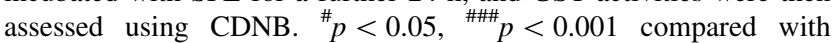
untreated control and $* * * p<0.001$ compared with Dox-treated control

417 were down-regulated) were differentially expressed with Dox treatment, and 2,015 genes (765 were up-regulated, 1,250 were down-regulated) were differentially expressed with Dox and SFE treatment. After DEGs were selected according to the criteria of a $>$ twofold change in expression and $p$ value $<0.05$, the DEGs were grouped into nine categories according to the pattern of gene expression: up-up, up-not changed (nc), up-down, nc-up, nc-nc, ncdown, down-up, down-nc, and down-down, based on the responses to Dox treatment, and Dox and SFE treatment. DEGs in the up-up category were up-regulated with Dox treatment compared with control, and up-regulated with Dox and SFE treatment compared with Dox treatment. Those in the up-nc category were up-regulated with Dox treatment compared with control, but showed no change with Dox and SFE treatment compared with Dox treatment. The genes in the up-up category (30), up-down category (0), nc-up category (29), and down-up category (2) were selected as the putative target DEGs involved in the cytoprotection of SFE against Dox-induced cytotoxicity. A total of 61 genes were selected and are listed in Table 1. These target DEGs were grouped into several categories on the basis of their gene ontologies and cellular functions, and included genes involved in glutathione metabolism and detoxification (such as GST theta 1, mu 1, and alpha type 2, and heme oxygenase 1), lipid, carbohydrate and energy metabolism (such as carnitine pamitoyltrasferase 1, transaldolase 1, and coenzyme Q6 homolog), protein metabolism, immune response (such as cytokine receptorlike factor 1 and integrin, beta 6), transmembrane transport (such as gap junction membrane channel protein alpha 4), chromosomal protein, apoptosis (such as cycline-dependent kinase inhibitor 1A), cell migration and motility, signal transduction and transcription.
Fig. 5 Diagrammatic representation of the genes tested. The figure shows how the genes were sorted on the basis of statistical criteria applied, as described in the "Methods." *Values in parentheses denote the number of probes with gene ontology

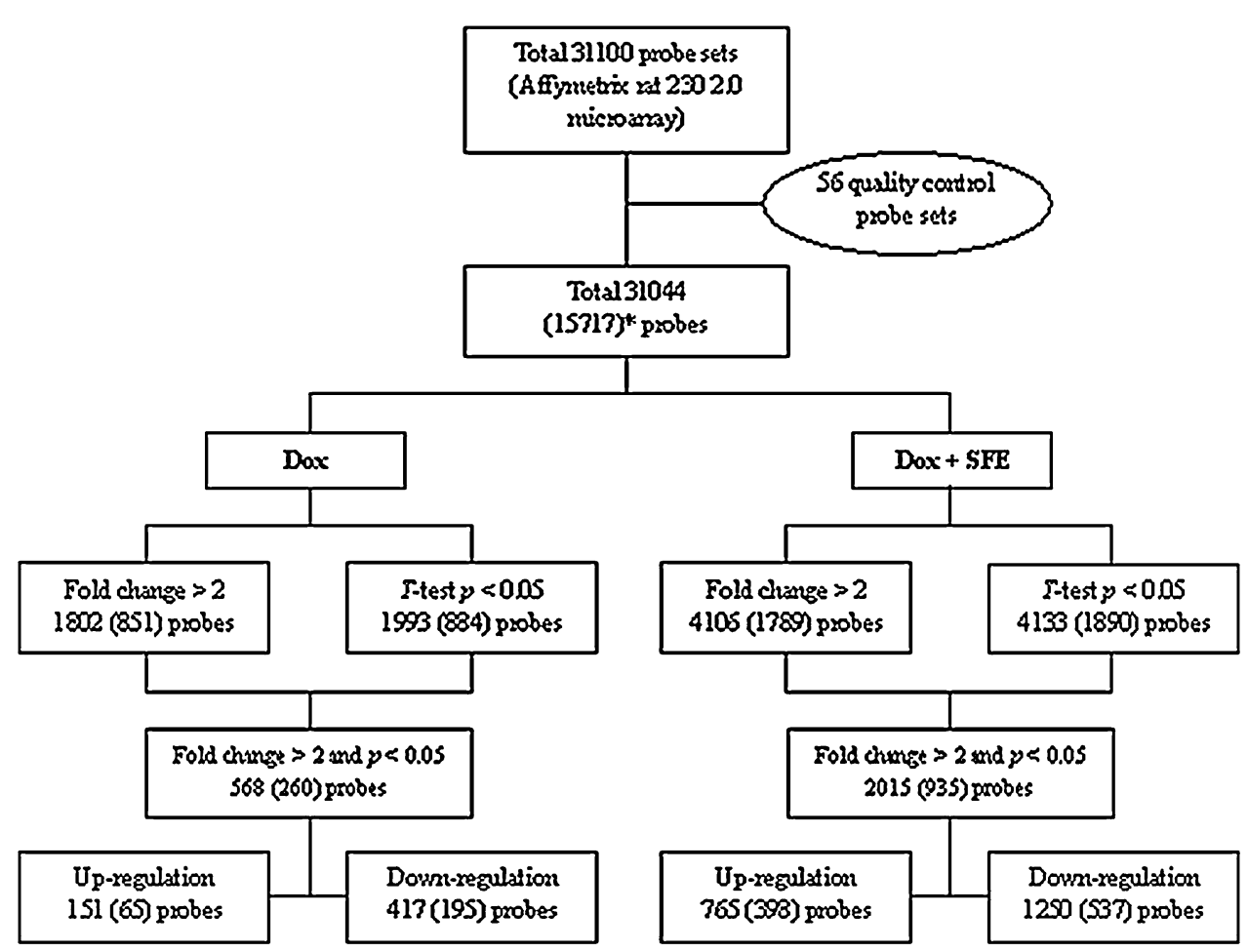


Table 1 Classified target genes implicated in cytoprotective effects of SFE on Dox-induced toxicity

\begin{tabular}{|c|c|c|}
\hline Accession no. & Description & Expression pattern $^{\mathrm{a}}$ \\
\hline \multicolumn{3}{|c|}{ Glutathione metabolism and detoxification } \\
\hline NM_053293 & Glutathione $S$-transferase theta 1 & up-up \\
\hline M28241 & Glutathione $S$-transferase, mu 1 & nc-up \\
\hline NM_017013 & Glutathione $S$-transferase, alpha type2 & up-up \\
\hline NM_053840 & Gamma-glutamyltransferase 1 & up-up \\
\hline NM_012580 & Heme oxygenase (decycling) 1 & up-up \\
\hline NM_012844 & Epoxide hydrolase 1, microsomal & nc-up \\
\hline \multicolumn{3}{|c|}{ Lipid metabolism } \\
\hline BM390364 & Transmembrane 7 superfamily member 2 & nc-up \\
\hline NM_031559 & Carnitine palmitoyltransferase 1 , liver & up-up \\
\hline U66322 & Leukotriene B4 12-hydroxydehydrogenase & up-up \\
\hline BI276935 & Dehydrogenase/reductase (SDR family) member 3 & nc-up \\
\hline BI293393 & Crystallin, lamda 1 & nc-up \\
\hline NM_133586 & Carboxylesterase 2 (intestine, liver) & up-up \\
\hline NM_012715 & Adrenomedullin & up-up \\
\hline AA996933 & CGI-58-like protein & up-up \\
\hline \multicolumn{3}{|c|}{ Carbohydrate metabolism } \\
\hline NM_031510 & Isocitrate dehydrogenase 1 (NADP+), soluble & nc-up \\
\hline NM_134407 & Aldo-keto reductase family 7, member A2 (aflatoxin aldehyde reductase) & nc-up \\
\hline NM_031972 & Aldehyde dehydrogenase family 3 , member A 1 & up-up \\
\hline NM_031811 & Transaldolase 1 & up-up \\
\hline NM_022592 & Transketolase & up-up \\
\hline \multicolumn{3}{|c|}{ Protein, amino acid metabolism } \\
\hline J02827 & Branched chain ketoacid dehydrogenase E1, alpha polypeptide & nc-up \\
\hline AI169359 & Cathepsin F & up-up \\
\hline \multicolumn{3}{|c|}{ Transmembrane and transport } \\
\hline NM_021654 & gap junction membrane channel protein alpha 4 & nc-up \\
\hline AB043636 & Potassium inwardly-rectifying channel, subfamily $\mathrm{J}$, member 8 & nc-up \\
\hline $\mathrm{BI} 285135$ & Similar to DNA segment, Chr 11, ERTO Doi 18, expressed & nc-up \\
\hline AI235230 & Potassium channel tetramerisation domain containing 13 & up-up \\
\hline AW915385 & Golgi associated, gamma adaptin ear containing, ARF binding protein 2 & nc-up \\
\hline NM_133315 & Solute carrier family 39 (iron-regulated transporter), member 1 & up-up \\
\hline BM386498 & Vacuolar protein sorting 33a (yeast) & nc-up \\
\hline NM_017154 & Xanthine dehydrogenase & nc-up \\
\hline \multicolumn{3}{|c|}{ Immune response } \\
\hline AA866388 & Cytokine receptor-like factor 1 (predicted) & up-up \\
\hline AF253065 & Chemokine-like factor 1 & nc-up \\
\hline AA925583 & B-cell linker & nc-up \\
\hline AI176519 & Immediate early response 3 & nc-up \\
\hline NM_030854 & Leukocyte cell derived chemotaxin 1 & nc-up \\
\hline AI070686 & Integrin, beta 6 & down-up \\
\hline \multicolumn{3}{|c|}{ Electron transport, energy metabolism } \\
\hline AI170570 & Coenzyme Q6 homolog (yeast) & up-up \\
\hline AI179227 & Similar to hypothetical protein MGC20446 & nc-up \\
\hline AI501131 & Lactate dehydrogenase $\mathrm{D}$ & nc-up \\
\hline \multicolumn{3}{|c|}{ Chromosomal protein } \\
\hline NM_022686 & Germinal histone $\mathrm{H} 4$ gene & up-up \\
\hline
\end{tabular}


Table 1 continued

\begin{tabular}{|c|c|c|}
\hline Accession no. & Description & Expression pattern $^{\mathrm{a}}$ \\
\hline AI235236 & Histone 2, Hh2aa (predicted) & up-up \\
\hline \multicolumn{3}{|l|}{ Apoptosis } \\
\hline U24174 & Cyclin-dependent kinase inhibitor $1 \mathrm{~A}$ & nc-up \\
\hline AA818262 & Angiopoietin-like protein 4 & up-up \\
\hline \multicolumn{3}{|c|}{ Cell migration, cell motility, localization of cell } \\
\hline AI146115 & EPH receptor B1 & nc-up \\
\hline NM_053843 & Fc receptor, Igg, low affinity III & up-up \\
\hline AI112954 & $A B I$ gene family, member 3 & nc-up \\
\hline \multicolumn{3}{|c|}{ Signal transduction } \\
\hline $\mathrm{AB} 004454$ & Presenilin 2 & up-up \\
\hline NM_012580 & Heme oxygenase (decycling) 1 & up-up \\
\hline \multicolumn{3}{|l|}{ Transcription } \\
\hline BF403357 & hpaII tiny fragments locus 9C & nc-up \\
\hline \multicolumn{3}{|l|}{ Others } \\
\hline AY081218 & Pregnancy-induced growth inhibitor & up-up \\
\hline BF403568 & UDP- $N$-acteylglucosamine pyrophosphorylase 1 -like 1 (predicted) & up-up \\
\hline AI059015 & Ectonucleoside triphosphate diphosphohydrolase 5 & up-up \\
\hline BI278288 & Similar to RIKEN cDNA 0610039C21 (predicted) & up-up \\
\hline AI235236 & Similar to Hist2h2aal protein & up-up \\
\hline BF416240 & Hypothetical LOC300751 (predicted) & nc-up \\
\hline BG375890 & Similar to hypothetical protein & nc-up \\
\hline BI295064 & Similar to brain protein 17 & nc-up \\
\hline AI411088 & Similar to RIKEN cDNA $4930570 \mathrm{c} 03$ & up-up \\
\hline$\perp \mathrm{BI} 296172$ & Similar to 2310010 G13Rik protein & up-up \\
\hline AI012782 & $\begin{array}{l}\text { Similar to cell surface receptor FDFACT, similar to paired } \\
\text { immunoglobin-like type } 2 \text { receptor beta }\end{array}$ & nc-up \\
\hline BI294552 & Similar to RIKEN cDNA 2310011J03 & up-up \\
\hline AI171553 & Similar to RIKEN cDNA 6330406115 (predicted) & down-up \\
\hline AI548652 & Similar to hypothetical $55.1 \mathrm{kDa}$ protein F09G8.5 in chromosome III & nc-up \\
\hline
\end{tabular}

a Among DEGs grouped into nine categories based on the pattern of gene expression, three categories, up-up, not changed (nc)-up, and downup, that were considered to be implicated in cytoprotective effects of SFE on Dox-induced toxicity were selected

\section{Discussion}

In the present study, Dox treatment significantly increased intracellular ROS production, lipid peroxidation and cytotoxicity in $\mathrm{H} 9 \mathrm{c} 2$ cardiomyocytes. The addition of SFE-H9c2 cells provided significant cytoprotection against Dox-induced cytotoxicity and reduced in intracellular ROS and lipid peroxidation.

Aerobic organisms have a multi-tiered defense system to combat oxidative stress, which provides protection not only against ROS, but also against the toxic electrophilic compounds generated by the interaction of ROS with cellular constituents, particularly the lipid peroxidation products. Thus, ROS scavenging is the first line of defense and inactivation of lipid peroxides is the second line of defense. In this study, we found that SFE also directly scavenged the intracellular ROS as the fisrt line of defense and reduced lipid peroxidation as the second line of defense, thereby protecting against Dox-induced cytotoxicity.

The functional classification of target DEGs could provide some clues to the cytoprotective mechanisms of SFE against Dox-induced cytotoxicity. Glutathione metabolism and detoxification-related genes were considered as the primary target genes involved in the cytoprotective action of SFE. GSTs are a large and diverse group of phase II biotransformation enzymes that function in the detoxification of xenobiotics and endogenous toxicants. The compounds detoxified by GSTs include a broad range of carcinogens, anticancer drugs, metabolic byproducts, as well as environmental chemicals. Some GST isozymes, especially alpha class GSTs, can efficiently reduce fatty acid hydroperoxides as well as phospholipid hydroperoxides, and can disrupt the autocatalytic chain of lipid peroxidation by reducing these hydroperoxides that 
propagate lipid peroxidation chain reactions [10]. Furthermore, GST alpha 1 and alpha 2 can use membrane phospholipid hydroperoxides as substrates in situ and can protect cell membranes at the sites of damage. The protective role of human GST alpha 2-2 (hGSTA 2-2) against oxidant toxicity has been demonstrated because transfection of K562 cells with hGSTA 2-2 protects these cells from $\mathrm{H}_{2} \mathrm{O}_{2}$ cytotoxicity [25].

Our microarray results showed that GST theta 1, mu 1, and alpha type 2 genes up-regulated by treatment with Dox and SFE compared with Dox treatment alone, as shown by the levels of cellular GST activity. Recently, it was reported that induction of cellular antioxidants and phase II enzymes, including GST alpha 1 , mu 1 , and pi 1 , by $3 \mathrm{H}-1$, 2-dithiole-3-thione (D3T) provided marked protection against $\mathrm{H} 9 \mathrm{c} 2$ cell injury caused by various oxidants and simulated ischemia-reperfusion [1].

In addition, one of the phase II enzymes, heme oxygenase 1 (HO-1), which catalyzes the degradation of heme to release free iron, carbon monoxide and biliverdin, is up-regulated. Some reports have demonstrated the potent antioxidative and cytoprotective properties of hemederived metabolites generated by HO-1 against various stress stimuli [26]. Microsomal epoxide hydrolase (EHm), which is another phase II enzyme and catalyzes the addition of $\mathrm{H}_{2} \mathrm{O}$ across the epoxide to form the corresponding diol [15], was also up-regulated by Dox and SFE treatment. These up-regulated cellular detoxifying systems might increase resistance to cell injury caused by Dox.

Many of the target DEGs that are up-regulated with Dox and SFE treatment are involved in lipid, carbohydrate and energy metabolism. Carnitine palmitoyltransferase-1 (CPT1), which is an integral outer mitochondrial membrane protein and the key regulatory enzyme of fatty acid oxidation, was up-regulated by SFE; long-chain fatty acids are the major substrates for energy production in the aerobic adult myocardium [18]. Transaldolase and transketolase also were up-regulated with SFE treatment. These enzymes are involved in the pentose phosphate pathway (PPP), which produces NADPH as a reducing equivalent for biosynthetic reactions and ribose 5-phosphate for the synthesis of nucleotides, RNA and DNA. This up-regulation of lipid, carbohydrate and energy metabolism-related genes can be partially helpful to the cytoprotective action of SFE, because the heart has high energy demands for maintenance of cellular processes, and abnormalities in the metabolic pathway that reduce energy production, transfer and utilization is one cause of heart failure [22].

In conclusion, this study demonstrates that SFE reduced Dox-induced cytotoxicity and intracellular ROS and lipid peroxidation, and increased cellular GST activity in H9c2 cardiomyocytes. The cDNA microarray data showed that SFE treatment modulates the gene expression of phase II detoxification enzymes such as GSTs, HO-1 and EHm, indicating that SFE might increase the resistance to cardiac cell injury by Dox via induction of the cellular detoxifying system. Future work will be needed to confirm our array results and to determine the functional role of these genes in the reduction of Dox toxicity.

Acknowledgments This work was supported by a research project on Functional Food Development from the Office for Government Policy Coordination, and by research grants from the Korea Institute of Science and Technology Evaluation and Planning (KISTEP) for functional food research and development, Ministry of Science and Technology, in the Republic of Korea.

\section{References}

1. Cao Z, Zhu H, Zhang L, Zhao X, Zweier JL, Li Y (2006) Antioxidants and phase 2 enzymes in cardiomyocytes: chemical inducibility and chemoprotection against oxidant and simulated ischemia-reperfusion injury. Exp Biol Med 231:1353-1364

2. Choi EH, Chang H-J, Cho JY, Chun HS (2007) Cytoprotective effect of anthocyanins against doxorubicin-induced toxicity in $\mathrm{H} 9 \mathrm{c} 2$ cardiomyocytes in relation to their antioxidant activities. Food Chem Toxicol 45:1873-1881

3. Choi EH, Ok HE, Yoon Y, Magnuson BA, Kim MK, Chun HS (2007) Protective effect of anthocyanin-rich extract from bilberry (Vaccinium myrtillus L.) against myelotoxicity induced by 5-fluorouracil. Biofactors 29:55-65

4. Doroshow JH (1991) Doxorubicin-induced cardiac toxicity. N Engl J Med 324:843-845

5. Doroshow JH, Locker GY, Myers CE (1980) Enzymatic defenses of the mouse heart against reactive oxygen metabolites: alterations produced by doxorubicin. J Clin Invest 65:128-135

6. Gille L, Nohl H (1997) Analyses of the molecular mechanism of adriamycin-induced cardiotoxicity. Free Radic Biol Med 23:775782

7. Goodman J, Hochstein P (1977) Generation of free radicals and lipid peroxidation by redox cycling of adriamycin and daunomycin. Biochem Biophys Res Commun 77:797-803

8. Habig WH, Pabst MJ, Jakoby WB (1974) Glutathione $S$-transferases. The first enzymatic step in mercapturic acid formation. J Biol Chem 249:7130-7139

9. Hancke JL, Burgos RA, Ahumada F (1999) Schisandra chinensis (Turcz.) Baill. Fitoterapia 70:451-471

10. Hayes JD, Flanagan JU, Jowsey IR (2005) Glutathione transferases. Annu Rev Pharmacol Toxicol 45:51-88

11. Hikino H, Kiso Y (1988) Natural products and liver disease. In: Wagner H, Hikino H, Farnsworth NR (eds) Economic and medicinal plant research. Academic Press Ltd, New York, pp 39-72

12. Ip SP, Mak DH, Li PC, Poon MK, Ko KM (1996) Effect of a lignan-enriched extract of Schisandra chinensis on aflatoxin $\mathrm{B}_{1}$ and cadmium chloride-induced hepatotoxicity in rats. Pharmacol Toxicol 78:413-416

13. Jo C, Ahn DU (1998) Fluorometric analysis of 2-thiobarbituric acid reactive substances in turkey. Poult Sci 77:475-480

14. Kang OH, Chae H-S, Choi J-H, Choi HJ, Park PS, Cho SH, Lee G-H, SO H-Y, Choo YK, Kweon O-H, Kwon D-Y (2006) Effects of the Schisandra fructus water extract on cytokine release from a human mast cell line. J Med Food 9:480-486 
15. Lu AY, Miwa GT (1980) Molecular properties and biological functions of microsomal epoxide hydrase. Annu Rev Pharmacol Toxicol 20:513-531

16. Lu H, Liu GT (1992) Anti-oxidant activity dibenzocyclooctene lingans isolated from Schisandraceae. Planta Med 58:311-313

17. Minotti G, Cairo G, Monti E (1999) Role of iron in anthracycline cardiotoxicity: new tunes for an old song? FASEB J 13:199-212

18. Neeley JR, Morgan HE (1974) Relationship between carbohydrate and lipid metabolism and the energy balance of heart muscle. Annu Rev Physiol 36:413-459

19. Nomura M, Ohtaki Y, Hida T, Aizawq T, Wakita H, Miyamoto K (1994) Inhibition of early 3-methyl-4-dimethylaminoazobenzeneinduced hepatocarcinogenesis by gomisin A in rats. Anticancer Res 14:1967-1971

20. Singal PK, Iliskovic N (1998) Doxorubicin-induced cardiomyopathy. N Engl J Med 339:900-905

21. Skehan P, Storeng R, Scudiero D, Monks A, McMahon J, Vistica D, Warren JT, Bokesch H, Kenney S, Boyd MR (1990) New colorimetric cytotoxicity assay for anticancer-drug screening. J Natl Cancer Inst 82:1107-1112

22. Ventura-Clapier R, Garnier A, Veksler V (2003) Energy metabolism in heart failure. J Physiol 555:1-13
23. Von Hoff DD, Layard MW, Basa P, Davis HL, Von Hoff AL, Rozencweig M, Muggia FM (1979) Risk factors for doxorubicininduced congestive heart failure. Ann Intern Med 91:710-717

24. Wang H, Joseph JA (1999) Quantifying cellular oxidative stress by dichlorofluorescein assay using microplate reader. Free Radic Biol Med 27:612-616

25. Yang Y, Cheng JZ, Singhal SS, Saini M, Pandya U, Awasthi S, Awasthi YC (2001) Role of glutathione $S$-transferases in protection against lipid peroxidation. Overexpression of hGSTA2-2 in $\mathrm{K} 562$ cells protects against hydrogen peroxide-induced apoptosis and inhibits JNK and caspase 3 activation. J Biol Chem 276:19220-19230

26. Zhang X, Shan P, Otterbein LE, Alam J, Flavell RA, Davis RJ, Choi AM, Lee PJ (2003) Carbon monoxide inhibition of apoptosis during ischemia-reperfusion lung injury is dependent on the p38 mitogen-activated protein kinase pathway and involves caspase 3. J Biol Chem 278:1248-1258

27. Zhu M, Lin KF, Yeung RY, Li RC (1999) Evaluation of the protective effects of Schisandra chinensis on phase I drug metabolism using a $\mathrm{CCl}_{4}$ intoxication model. J Ethnopharmacol 67:61-68 